\title{
Type 1 Diabetes and Attention Deficit Hyperactivity Disorder: Development of New Educational Material
}

\author{
Sofia Sjöberg (Sofia.K.Sjoberg@regionostergotland.se), Magnus Edgren and Kerstin Gutefeldt \\ Department of Endocrinology and Department of Medical and Health Sciences, Linköping University, Linköping Sweden.
}

\section{Background}

Patients with type 1 diabetes and Attention Deficit Hyperactivity Disorder (ADHD), fail to attend from clinic al visits more often than patients with without ADHD. If they come to the visit, they are often late, plasma glucose self-monitoring meters are absent, blood specimen collections are frequently forgotten and difficulties in managing self-care are noticed. Decreased ability of planning, perception of time, impulse control, setting goals, ability of organizing actions over time and a decreased rewarding system are the core symptom of ADHD $(1,2)$. In individuals diagnosed with ADHD, correlations between decreased executive functions and poor diabetes control has been shown (3).

\section{Aims}

Primary: To develop education materials adapted for patients with type 1 diabetes and ADHD, aiming to improve the understanding and knowledge of their personal diabetes care.

Secondary: To develop information material about ADHD and diabetes useful to staff at the diabetes clinic.

Table 1. Summary of information booklet for staff

- Highlight date and time in letter of invitation . Send sms to announce the visit.

- Minimize distractions: Clear your desktop, close the window and turn off the phone.

- Write down an agenda, point by point for the visit. Choose a few topics, minimising the risk for patient loosing concentration.

- Use flow chart and pictures.

- Encourage and give positive feedback. Negative feedback will increases the risk of decreased motivation

- Go through diabetic tools, insulin regime and reminders.

- Consider if collaboration with a psychiatric or habilitation clinic could be of use for your patient.

- Summarize the session to reassure the patient has got the message. Hand out memory notes or let the patient take a photo of the notes.

We thank C. Nylander, pediatrician and $\mathrm{PhD}$ student at the County hospital in Eskilstuna and the department of Woman and Children's Health, Uppsala

University, for reviewing the educational material.

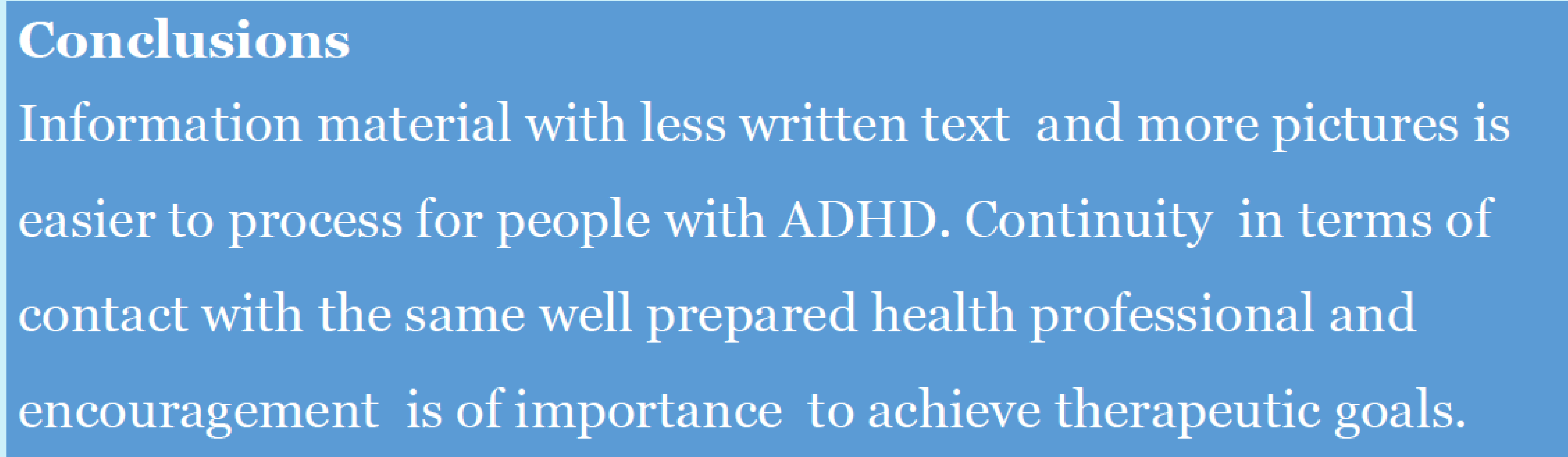

Fig 1, 2. Flow charts illustrating relations and actions for high and low blood glucose levels.
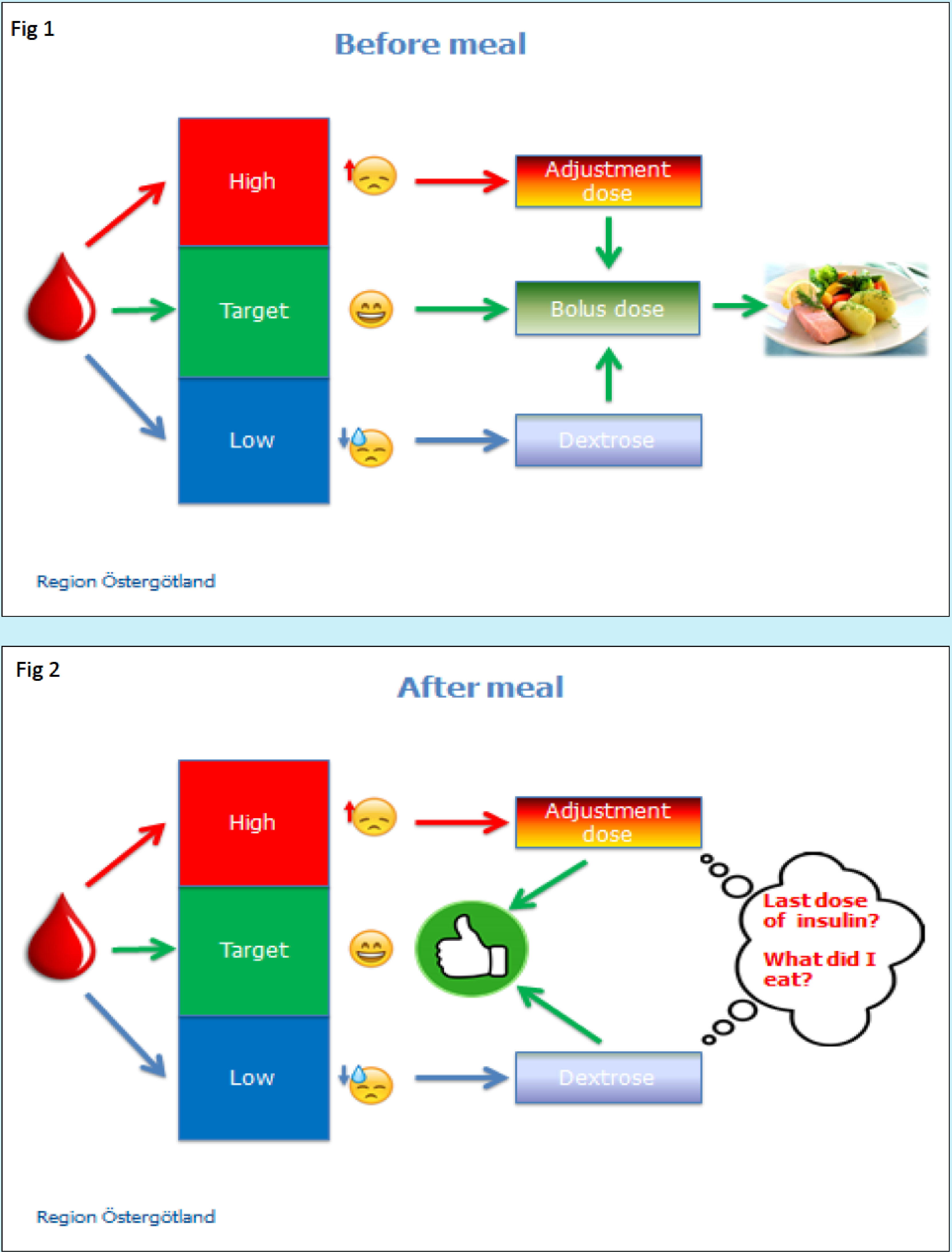

\section{Results}

Education material with pictures for patients has been produced (fig 1, 2). An information booklet for staff about ADHD has been written (table 1). Comments from three of our patients:

"For me, using pictures is the only way of learning".

"Information in text gets confusing, I don't read it. The flow charts are good reminders how to think about meals and doses".

"It is practical to follow the arrows in the flow chart, I know what to do".

References

1. Hjärnfonden. Swedish Brain Foundation

2. R. Stark. E. Bauer et. Al. 2010 Bender Institute of Nuroimagin 3.C. Nylander et. al 2012 Acta Pediatrica 